\title{
Antimicrobial defensin peptides of the human ocular surface
}

Richard John Haynes, Patrick Jason Tighe, Harminder Singh Dua

\begin{abstract}
Backgroundlaims-The antimicrobial activity of the tear film exceeds the activity of its known constituents. The authors postulate that this excess activity is the result of antimicrobial peptides called defensins, and they aimed to look for defensins in the human eye.

Methods-Evidence of defensin production was sought by reverse transcriptase polymerase chain reaction (RT-PCR). Intron spanning primers were designed for $\beta$ defensins 1 and 2 , and $\alpha$ defensins 5 and 6 . RT-PCR was performed on cornea, conjunctiva, and lacrimal gland samples, and reaction products were size fractionated and sequenced to confirm their identity. A monoclonal antibody was utilised for the detection of $\alpha$ defensins 1,2 , and 3 in tissue sections and in immunoblots of tears.

Results-RT-PCR revealed $\beta$ defensin 1 message in samples of conjunctiva, cornea, and lacrimal gland. $\beta$ Defensin 2 message was detected in the conjunctiva and cornea but was absent from the lacrimal gland. $\alpha$ Defensin 5 and 6 message was absent in these tissues but $\alpha$ defensins 1,2 , and 3 were detected in normal tears, lacrimal gland, and inflamed conjunctiva by immunochemistry.

Conclusion-The data suggest the human eye innately produces a spectrum of antimicrobial defensin peptides. Defensins hold therapeutic potential in ocular infections as they have a broad spectrum of antimicrobial activity (bacteria fungi and viruses ) and accelerate epithelial healing. (Br F Ophthalmol 1999;83:737-741)
\end{abstract}

Laboratory for Eye Research, University of Nottingham and

Department of Ophthalmology, Queen's Medical Centre University Hospital, Nottingham R J Haynes H S Dua

Larry A Donoso Laboratory for Eye Research, University of Nottingham and Departments of Ophthalmology and Immunology, Queen's Medical Centre,

University Hospital

Nottingham

P J Tighe

Correspondence to: Professor H S Dua.
Defensins are naturally occurring peptides that are considered to be among the earliest developed molecular effectors of innate immunity. ${ }^{2}$ They are highly conserved molecules being present in many animal classes (mammals, birds, insects, and amphibians). ${ }^{3}$ Defensins have a wide range of antimicrobial activity encompassing Gram positive and Gram negative bacteria, fungi, and viruses (including HIV and HSV). ${ }^{4-6}$ They are also believed to accelerate wound healing by virtue of their mitogenic effect on epithelial cells and fibroblast. ${ }^{7}$

Two families of mammalian defensins, $\alpha$ and $\beta$, have been described. In humans, $\alpha$ defensins are largely present in neutrophils ( $\alpha$ defensins $1-4)^{89}$ and in the small intestinal Paneth cells ( $\alpha$ defensins 5 and 6$).^{10-12} \beta$ Defensins have a wider cellular distribution than $\alpha$ defensins, $\beta$ defensin 1 being expressed by the pancreas, kidney, and respiratory epithelium. ${ }^{13}$ Recently $\beta$ defensin 2 has been demonstrated in the skin and bronchial mucosa. ${ }^{14}$ No defensin has yet been isolated from any ocular tissue in any species.

A study was undertaken to look for evidence of the known human defensin subclasses in the eye and identify the cells responsible for their production. The aim of the experiments was to characterise tissues in terms of the different classes of defensins they produce.

\section{Materials and methods}

OCULAR SAMPLE PREPARATION

Samples of human cornea, conjunctiva, and lacrimal gland were taken from cadaveric eyes donated and consented for transplantation and research. The ocular tissue samples analysed included four corneal, eight conjunctival, and two lacrimal gland. Positive control samples analysed included two samples from cultured skin cells and two samples from growth arrested bronchial cell cultures. Negative control samples analysed (for $\beta$ defensin polymerase chain reaction (PCR)) included one sample of neutrophils from peripheral blood and one retinal sample. The neutrophil sample also served as a positive control for $\alpha$ defensins 1,2 , and 3 immunohistology.

Samples were collected for both reverse transcriptase polymerase chain reaction (RTPCR) and immunohistochemical analysis. Antibodies to $\beta$ defensins 1 and 2 and $\alpha$ defensins 5 and 6 are currently not available, therefore evidence of production of these defensins was sought by RT-PCR. The corneal and conjunctival epithelial cell samples for RT-PCR were collected by scraping the epithelial surfaces with a scalpel blade and suspending the collected cells in saline by agitating the blade in saline. The samples were then 
transported to the laboratory, where they were centrifuged, and the cell pellet treated with lysis buffer for total RNA extraction. Samples of lacrimal gland tissue, $2 \mathrm{~mm} \times 2 \mathrm{~mm}$, were ground up under liquid nitrogen with a mortar and pestle and treated with lysis buffer for total RNA extraction.

Evidence of $\alpha$ defensins 1, 2, and 3 production was sought by immunohistochemistry in both paraffin and cryo sections of ocular tissue samples. Samples were fixed in formalin for paraffin section preparation. Cryo samples were transported to the laboratory in saline and immediately oriented on a piece of cucumber, ${ }^{15}$ immersed in OCT, and snap frozen in liquid nitrogen before cryosectioning to $6 \mu \mathrm{m}$ thickness on a Leica CM 1900 cryostat.

$\alpha$ Defensins 1, 2, and 3 production was also analysed by immunoblotting tear samples collected from healthy volunteers, and aqueous humour samples collected from patients undergoing cataract surgery. Appropriate consent was obtained from all individuals.

Neutrophils were purified from citrated whole blood using Polymorphprep (Nycomed Pharma AS) as in manufacturer's instructions. The neutrophils were then fixed to slides by cytospinning and used as positive controls during immunochemistry (neutrophils contain large amounts of $\alpha$ defensins 1, 2, and 3).

TOTAL RNA PURIFICATION AND C-DNA SYNTHESIS Total RNA was purified from the fresh cell samples using the RNEASY Total RNA Kit (Qiagen Inc, Chatsworth, CA, USA) as in manufacturer's instructions.

c-DNA was synthesised directly from total RNA using the "Ready to go" T-Primed First Strand Kit (Pharmacia Biotech) as in manufacturer's instructions. Adequate c-DNA synthesis in each sample was confirmed by employing a hypoxanthine phosphoribosyl transferase (HPRT) PCR reaction. HPRT is a low level message produced by all living cells, and will be detected by PCR if an adequate amount of c-DNA is present in the sample.

PCR

Published $\beta$ defensin 1 and 2 and $\alpha$ defensin 5 and 6 genomic DNA and messenger RNA sequences were used to design primers for RT-PCR analysis ${ }^{16}$ (Gen-Bank database accession numbers X92744, U50930, U50931, Z71389, M96679, M96682, M97925). $\beta$ Defensin 1 primers were designed to span the entire intron of $\beta$ defensin 1 DNA, thus making contaminant products derived from genomic DNA readily detectable.

$\beta$ defensin 2 primers were based on the m-RNA sequences as the genomic DNA sequence is not currently known. These primers failed to produce an appropriate product when tested with pure genomic DNA, suggesting the primers span an intron. A $\beta$ defensin 2 RT-PCR product of the correct size must therefore be derived from the messenger RNA and not genomic DNA. $\alpha$ Defensin 5 and 6 intron spanning primers were designed on the basis of published sequences.
c-DNA $(0.5 \mu \mathrm{l})$ was added to a mixture (final volume $25 \mu \mathrm{l}$ ) that contained $0.5 \mu \mathrm{l}$ dNTP mix, $0.25 \mu 11 \%$ Tween, $0.5 \mu 1$ of both 3' and $5^{\prime}$ primers $(20 \mu \mathrm{mol} / \mu \mathrm{l}), 1 \mu \mathrm{l}$ of Elongase Enzyme Mix (Taq and Pyrococcus species GB-D thermostable DNA polymerases), $5.0 \mu \mathrm{l} 5 \mathrm{x}$ Buffer B and $16.75 \mu 1$ DEPC water. AmphiTaq Gold DNA polymerase was utilised in $\alpha$ defensin 5 and 6 PCR reactions. PCR amplification was performed with an automated thermal cycler for 30 cycles. $\beta$ defensin PCR products were sequenced by an ABI Prism DNA sequencer.

\section{IMMUNOBLOTTING}

DEF 3 (Bachem UK Ltd), the only antidefensin monoclonal antibody available to date, was used for dot blotting, western blotting, and immunohistochemistry. DEF 3 is a cross reactive antibody to $\alpha$ defensins 1,2 , and 3.

Five tear and three aqueous humour samples were dot blotted onto a nitrocellulose membrane and allowed to dry. DEF 3, 1:500, was used as the primary antibody. The secondary antibody, was a rabbit anti-mouse (Dako, 1:1000) and the tertiary antibody was mouse APAAP (alkaline phosphatase antialkaline phosphatase, Dako). TRIS buffered saline 0.05 $\mathrm{M}, \mathrm{pH} 7.6$ was the wash buffer between each antibody step and BCIP/NBT (Sigma Fast) was the substrate. Endogenous alkaline phosphatase activity was blocked with levamisole. Negative control samples, where the primary antibody was replaced with wash buffer only, were run simultaneously.

Four tear samples also underwent sodium dodecyl sulphate polyacrylamide gel electrophoresis (SDS-PAGE) and western blotting. During the preparation of tear samples for SDS-PAGE, the tears were reduced and boiled before electrophoresis. The effect of the boiling step on epitope antigenicity was assessed by also immunoblotting tear samples that had previously undergone boiling.

\section{IMMUNOHISTOCHEMISTRY}

Ocular tissue samples were analysed in both paraffin and cryo sections. Six normal and two pathologically inflamed conjunctival paraffin sections underwent standard immunohistochemistry using DEF 3 as the primary antibody, biotinylated rabbit anti-mouse antibody (Dako 1:500) as the secondary antibody and StreptABC Complex/HRP as the tertiary conjugate. Diaminobenzidine (Dako) was used as the substrate and $0.05 \mathrm{M}$ TRIS buffered saline $\mathrm{pH} 7.6$ was used as the wash buffer. Endogenous peroxidase activity was blocked with methanol +20 vol $(6 \%)$ hydrogen peroxide solution. Positive and negative controls were run simultaneously.

For lacrimal gland cryo samples a standard APAAP staining technique with fast red substrate (Sigma Fast) was used (using the antibodies described for immunoblotting above). 
A

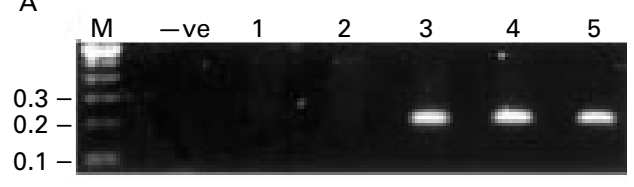

B

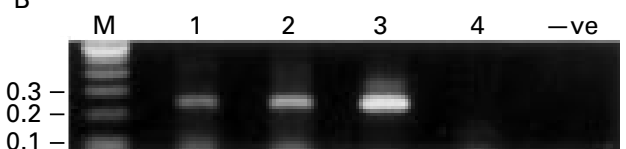

Figure 1 (A) Analysis of $\beta$ defensin 1 expression by RT-PCR. Lane $M=100$ bp ladder, lane -ve = negative control sample (DEPC $\mathrm{H}_{2} \mathrm{O}$ replacing $\left.c-D N A\right)$, lane $1=$ neutrophils, lane $2=$ retina, lane $3=$ conjunctiva, lane $4=$ cornea, lane $5=$ lacrimal gland. Bronchial cell positive control RT-PCR confirmed a product at $215 \mathrm{bp}$ (not shown). $\beta$ Defensin 1 primer sequence: 5'-CCC AGT TCC TGA AAT CCT GA-3'; 5'-CAG GTG CCT TGA ATT TTG GT-3'. Product size $=215$ bp. (B) Analysis of $\beta$ defensin 2 expression by RT-PCR. Lane $M=100$ bp ladder, lane 1=skin, lane 2=conjunctiva, lane 3=cornea, lane

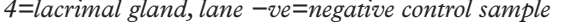
(DEPC $\mathrm{H}_{2} \mathrm{O}$ replacing c-DNA). $\beta$ Defensin 2 primer sequence: 5'-CCA GCC ATC AGC CAT GAG GGT-3'; 5'-GGA GCC CTT TCT GAA TCC GCA-3'. Product size $=255 \mathrm{bp}$.

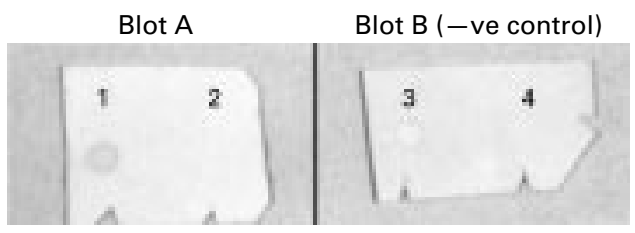

Figure 2 Dot immunoblots of normal human tears showing positive staining for a defensins 1-3. Blot A: immunostain with anti a defensin 1-3 monoclonal antibody; dot $1=$ tears, dot $2=$ non-defensin peptide mixture (negative control). Blot B (negative control): immunostain as blot $A$ except wash buffer replaced anti a defensin 1-3 monoclonal antibody; dot 3=tears, dot 4=non-defensin peptide mixture. APAAP stain.

\section{Results}

$\beta$ Defensin 1 RT-PCR of cornea, conjunctiva, and lacrimal gland revealed a product at 215 base pairs in all samples with adequate c-DNA yields, confirmed by HPRT RT-PCR (Fig 1A). Sequencing of this product revealed an almost exact match with the published sequence of the $\beta$ defensin 1 messenger RNA.

$\beta$ Defensin 2 RT-PCR revealed strong signals at 255 base pairs from all cornea samples and weaker signals from all conjunctival samples with adequate c-DNA yields confirmed by HPRT RT-PCR, but no product was detected from any of the lacrimal gland RT-PCR reactions (Fig 1B). This result parallels previous experiments which have shown $\beta$ defensin 1 but not $\beta$ defensin 2 production by the salivary gland, ${ }^{13}{ }^{14}$ a gland similar in structure to the lacrimal gland. (NB only two lacrimal glands were analysed but adequate c-DNA levels were demonstrated in these samples by the HPRT PCR and the presence of an appropriate product from the $\beta$ defensin 1 PCR reaction on the same samples.) Sequencing of the corneal RT-PCR product revealed an almost exact match with the published sequence of the $\beta$ defensin 2 messenger RNA.
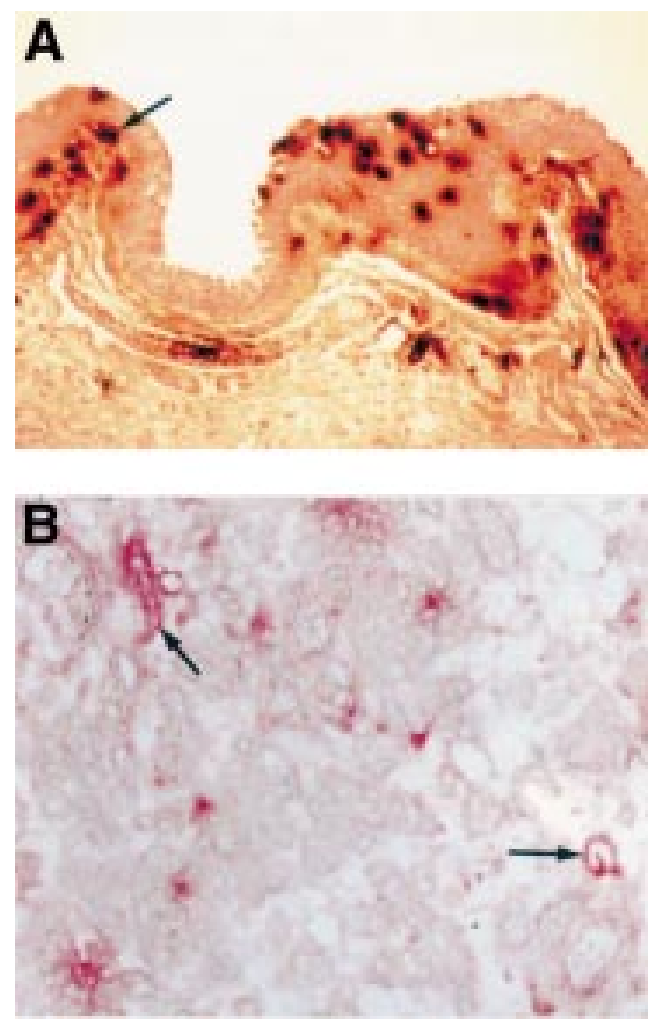

Figure 3 (A) Immunohistochemistry of inflamed conjunctiva showing positive staining for a defensins 1-3 in neutrophils in the conjunctival epithelium (arrow) and substantia propria (immunoperoxidase paraffin $\times 100$ ). (B) Immunohistochemistry of lacrimal gland showing positive staining for a defensins $1-3$ in the ductular epithelium (arrows) (APAAP cryosection $\times 100)$.

$\alpha$ Defensin 5 and 6 RT-PCR did not result in a PCR product in any of the ocular samples tested.

Dot immunoblots of all normal human tear samples tested showed positive staining with DEF 3 (Fig 2). This suggests that $\alpha$ defensins 1,2 , and 3 (either all or just one) are free in solution in the tears possibly released from neutrophils.

SDS-PAGE of normal tears revealed a peptide band of approximately double the molecular weight of defensin (defensins are known to form dimers); however, western blots of these peptides did not stain with DEF 3. This lack of western blot staining may be due to epitope denaturation during the boiling step of SDS-PAGE sample preparation. This theory is confirmed by the fact that dot immunoblots of positively staining tears failed to stain with DEF 3 after boiling, probably because of epitope destruction by boiling.

Only one out of three fresh undiluted aqueous humour dot blots was weakly positive at the concentrations tested.

Immunohistochemistry of the inflamed conjunctiva samples showed positive staining of masses of neutrophils in the conjunctival substantia propria and epithelium (Fig 3A); however, all of the normal uninflamed conjunctiva samples failed to stain with DEF 3. Immunohistochemistry of the lacrimal gland samples tested showed staining of the ductular epithelial cells with DEF 3 (Fig 3B) and high 
power views revealed staining of intracellular granules within the lining epithelial cells of the ductules.

\section{Discussion}

Our data suggest that the human eye innately produces a spectrum of antibiotic defensin peptides, and that examples from both $\alpha$ and $\beta$ defensin subclasses are present in the human eye. It appears that the $\beta$ defensins ( $\beta$ defensin 1 and $\beta$ defensin 2 ) are actually produced by the ocular surface and that $\alpha$ defensins 1,2 , and 3 are largely released into the ocular surface fluids by resident or passing neutrophils and possibly by secretion from lacrimal ductular epithelia. $\alpha$ Defensins 5 and 6 were not detected in the eye, thus on the information currently available, defensin 5 and 6 remain the sole product of small intestine Paneth cells.

These results represent the first data revealing defensin production by the eye of any species and implicate defensins in the innate protection of the human ocular surface.

All organs of the body that connect with the exterior are lined by mucus membrane. The mucus membrane of the eye is the conjunctiva, which together with the corneal epithelium, constitutes the ocular surface. The mucosa of the gut, respiratory, and urogenital tract collectively function as the mucosal immune system. ${ }^{17}$ Recent evidence indicates that the ocular surface is an integral part of the mucosal immune system. ${ }^{18-20}$ Like the skin, all mucosal surfaces of the body are in constant interaction with environmental micro-organisms and hence vulnerable to infection. The protection of the eyes from microbial attack therefore, must have been of paramount importance for the survival of the individual and the preservation of the species. Besides possessing several cell mediated defence modalities in common with other mucous membranes, the eye has developed, through evolution, a unique array of protective mechanisms. The preocular tear film contains secretory IgA and IgG, complement components, lactoferrins, lysozyme, $\beta$ lysins, orosomucoid, and caeruloplasmin which prevent bacterial adherence and also kill organisms. $^{21}$ The antimicrobial activity of tears, however, is known to be in excess of its known constituents. ${ }^{1}$ We postulated that a significant contribution to the ocular defence could come from innately produced antimicrobial peptides called defensins, and in this study we showed that this was indeed the case.

All data available thus far on the defensin molecules present at mucosal surfaces indicate an important role in defence against microbial invasion. An understanding of the exact mechanism of production and regulation of defensins at the ocular surface will provide further insight into occurrence of microbial corneal ulcers, which often leave behind sight threatening sequelae. Historically, it has been well established that breach of corneal epithelial integrity is the most important risk factor in corneal infection. This may be more than just a mechanical effect, with a break in the local defensin barrier also contributing to the susceptibility to infection.

Defensins are naturally occurring peptides that are considered to be among the earliest developed molecular effectors of innate immunity. ${ }^{2}$ They are highly conserved molecules being present in many animal classes (mammals, birds, insects, and amphibians). ${ }^{3}$ Defensins are short peptide molecules with a primary chain length of 29-35 amino acids and molecular weight of $3.5-4.5 \mathrm{kDa}$. They are cationic, variably arginine rich, and contain six conserved cysteine residues which form three intramolecular disulphide bonds that stabilise a rigid three dimensional $\beta$ sheet structure. ${ }^{22} 23$ They function as dimers by creating voltage sensitive channels in the plasma membrane of the target organism. ${ }^{23-26}$ The antimicrobial spectrum of defensins encompasses Gram positive and Gram negative bacteria, fungi, and viruses (including HIV and HSV)..$^{4-6}$ Defensins may also promote a rapid cellular immune response to infection via a chemotactic effect on monocytes. ${ }^{27}$ In addition to their antimicrobial actions defensins may accelerate wound healing, by virtue of their mitogenic effect on epithelial cells and fibroblasts. ${ }^{7}$

It is interesting to hypothesise that purified or recombinant defensins may be ideal therapeutic agents in the eye as they could be applied directly to the site of infection on the ocular surface. Of particular clinical interest is that defensins have a broad spectrum of activity, promote epithelial healing, and may be non-antigenic. This contrasts with most current antibiotics which have a comparatively limited spectrum of activity (for example, Gram negative bacteria only), interfere with healing as a result of ocular surface toxicity, and sometimes produce allergic reactions. Defensins may therefore offer a more refined approach to the management of ocular infections and may be effective in the treatment of established ocular infections or in the prevention of keratitis in eyes at particular risk such as dry eye states, contact lens wearers, critically ill or comatose patients, ${ }^{21}$ or after ophthalmic surgery where promotion of epithelial healing would be of benefit. Factors controlling the production of ocular defensins are unknown and it is likely that some of the infection risk factors mentioned above are associated with downregulation of defensin production.

The experiments described in this paper relied mainly on RT-PCR, as the recent discovery of defensins, and possibly their low antigenic potential, restricts the availability of anti-defensin antibodies (our attempt to make antibodies was unsuccessful). The precise cell population responsible for defensin production in the cornea, conjunctiva (that is, basal, suprabasal, or superficial), and lacrimal gland have not been identified, therefore future studies with in situ hybridisation and monoclonal antibodies, as and when they become available, will provide this useful information. Also, an evaluation of defensin production in ocular surface disease states that predispose to infection will provide a means of initiating a 
new approach in the management of these conditions.

We thank Professor Larry A Donoso, director of research, Wills We thank Professor Larry A Donoso, director of research, Wills
Eye Hospital, Philadelphia, USA, for providing defensin peptides.

1 Friedland BR, Anderson DR, Forster RK. Non-lysozyme antibacterial factor in human tears. Am $\mathcal{F}$ Ophthalmol antibacterial

2 Zasloff $M$. Antibiotic peptides as mediators of innate immunity. Curr Opin Immunol 1992;4:3-7.

3 Martin E, Ganz T, Lehrer RI. Defensins and other endogenous peptide antibiotics of vertebrates. F Leukoc Biol 1995;58: 128

4 Lehrer RI, Lichtenstein AK, Ganz T. Defensins: antimicrobial and cytotoxic peptides of mammalian cells. Annu Rev Immunol 1993;11:105-28.

5 Daher KA, Selsted ME, Lehrer RI. Direct inactivation of viruses by human granulocyte defensins. F Virol 1986;60: 1068-74.

6 Nakashima H, Yamamoto N, Masuda M, et al. Defensins inhibit HIV replication in Vitro. AIDS 1993;7:1129.

7 Murphy CJ, Foster BA, Mannis MJ, et al. Defensins are mitogenic for epithelial cells and fibroblasts. $\mathcal{F}$ Cell Physiol 1993;155:408-13.

8 Ganz T, Selsted ME, Szklarek D, et al. Defensins: natural peptide antibiotics of human neutrophils. $\mathcal{f}$ Clin Invest 1985;76:1427-35.

9 Ganz T, Lehrer RI. Defensins, Pharmacol Ther 1995;66: Ganz T ,

10 Selsted ME, Miller SI, Henschen AH, et al. Enteric defensins. Antibiotic peptide components of intestinal host defence. F Cell Biol 1992;118:929.

11 Jones DE, Bevins CL. Paneth cells of the human small intestine express an antimicrobial peptide gene. 7 Biol Chem 1992;267:23216-25.

12 Jones DE, Bevins CL. Defensin-6 m-RNA in human Paneth cells: implications for antimicrobial peptides in the host defense of the human bowel. FEBS Lett 1993;315:187-92

13 Zhao C, Wang I, Lehrer RI. Widespread expression of betadefensin hBD-1 in human secretory glands and epithelial cells. FEBS Lett 1996;396:319-22.
14 Harder J, Bartels J, Christophers E, et al. A peptide Harder J, Bartels J, Christophers E, et al. A
antibiotic from human skin. Nature 1997;387:861.

15 Dua HS, Gomes JAP, Singh A, et al. Fresh-frozen cucumber as a mount for conjunctival and corneal tissue in cryomicrotomy. Arch Ophthalmol 1994;112:1139-41.

16 Liu L, Zhao C, Heng HH, et al. The human beta-defensin-1 and alpha-defensins are encoded by adjacent genes: two peptide families with differing disulfide topology share a common ancestry. Genomics 1997;43:316-20.

17 Strober W, James SP. The mucosal immune system. In: Stites DP, Terr AI, eds. Basic and clinical immunology. 7th ed. New York: Appleton and Lange, Prentice-Hall ed. New York: Appleton

18 Dua HS, Gomes JAP, Donoso LA, et al. The ocular surface as part of the mucosal immune system: Conjunctival mucosa-specific lymphocytes in ocular surface pathology. Eye 1995;9:261-7.

19 Dua HS, Donoso LA, Laibson PR. Conjunctival instillation of retinal antigens induces tolerance. Does it invoke mucosal tolerance via conjunctiva associated lymphoid tissue (CALT)? Ocular Immunol Inflamm 1994;2:29-36.

20 Dua HS, Gomes JAP, Jindal VK, et al. Mucosa specific lymphocytes in the conjunctiva, corneoscleral limbus and phocytes in the conjunctiva, corneoscleral

21 Dua HS. Bacterial keratitis in the critically ill and comatose patient. Lancet 1998;351:381-8.

22 Lehrer RI, Ganz T, Selsted ME. Defensins: endogenous antibiotic peptides of animal cells. Cell 1991;64:229-30.

23 Hill CP, Yee J, Selsted ME, et al. Crystal structure of defensin HNP-3, an amphiphilic dimer. Mechanisms of membrane permeabilization. Science 1991;251:1481.

24 Lehrer RI, Barton A, Daher KA, et al. Interaction of human defensins with Escherichia coli. Mechanism of bactericidal activity. F Clin Invest 1989;84:553-61.

25 Kagan BL, Ganz T, Lehrer RI. Defensins: a family of antimicrobial and cytotoxic peptides. Toxicology 1994;87: 131-49.

26 Lohner K, Latal A, Lehrer RI, et al. Differential scanning microcalorimetry indicates that human defensin, HNP-2, interacts specifically with biomembrane mimetic systems. Biochemistry 1997;36:1525-31.

27 Territo MC, Ganz T, Selsted ME, et al. Monocytechemotactic activity of defensins from human neutrophils. f Clin Invest 1989;84:2017-20. 